\title{
Rheological behavior of wood in stress relaxation under compression
}

\author{
Sabina Huč ${ }^{1,2}$ - Tomaž Hozjan ${ }^{3} \cdot$ Staffan Svensson $^{1}$
}

Received: 4 October 2017 / Published online: 9 February 2018

(C) The Author(s) 2018. This article is an open access publication

\begin{abstract}
Rheological behavior of wood under uniaxial compression along and perpendicular to the grain in constant environment was examined. Tests with constant deformation rate until failure and stress relaxation tests with constant deformation applied stepwise were carried out. The experimental results of stress relaxation showed nonlinear material behavior over time that got more prominent under high deformation levels. Considerable amount of stress relaxed during applying the deformation. Wood experienced greater stress relaxation along the grain than perpendicular to it. Three rheological models for orthotropic material were calibrated to the experimentally determined stress-time curves in longitudinal and transverse directions simultaneously. Small deformation levels assuming linear strains were accounted for in the models. Required elastic material parameters were determined from the tests with constant deformation rate. A model including the highest number of viscoelastic material parameters was the most successful in predicting stress relaxation of wood under stepwise deformation. Modeling indicated that wood behavior was very close to linear viscoelastic in relaxation under small deformation. The obtained material parameters made the model suitable for predicting rheological behavior of wood comprehensively, under sustained deformation or load in constant conditions.
\end{abstract}

Sabina Huč

sabina.huc@hb.se

1 Faculty of Textiles, Engineering and Business, University of Borås, 50190 Borås, Sweden

2 Ångström Laboratory, Division of Applied Mechanics, Uppsala University, 75121 Uppsala, Sweden

3 Faculty of Civil and Geodetic Engineering, University of Ljubljana, 1000 Ljubljana, Slovenia 


\section{Introduction}

Time-dependent material behavior is an important feature to be considered when wood is used as a structural material. Time-dependent behavior of wood strongly depends on temperature, moisture content level, history and variation, stress and deformation level, wood species, material heterogeneity, etc. In constant climate and nonzero loading or deformation state, the material behavior of wood is viscoelastic. When the moisture content in wood varies and the material is stressed or deformed simultaneously, its behavior is characterized as mechanosorptive. The phenomenon when material's deformation increases over time under constant stress is called creep. The opposite process when the deformation is held constant and stress reduction over time occurs is called relaxation. A complementary amount of stress that is maintained is called stress retention. Creep and relaxation processes trigger the same molecular mechanisms in wood (Engelund and Svensson 2011; Eitelberger et al. 2012). The relation between creep and stress relaxation phenomena is also mathematically supported in the theory of linear viscoelasticity. The derivation in onedimensional form can be found in many theoretical books on viscoelasticity (e.g., Findley et al. 1976; Flügge 1967). Mechanosorptive and viscoelastic creep are also considered as connected or as the same mechanisms (Hanhijärvi and Hunt 1998; Hanhijärvi 1995a, b; Hunt 1999; Bažant 1985). Rheological behavior of wood has been experimentally and theoretically studied for decades. A recent review on the topic has been made by Navi and Stanzl-Tschegg (2009). Evidently, many studies consider creep behavior of wood (e.g., Ożyhar et al. 2013; Schniewind and Barrett 1972; Taniguchi and Ando 2010; Kawahara et al. 2015), while the experimental research on stress relaxation is very limited. This is somehow puzzling, since it has been established that wood experiences rheological behavior whether the deformation or the stress is held constant; meaning that the rheological behavior of wood is characterized by both creep of strain and stress relaxation phenomena. Therefore, the importance of research on stress relaxation equals the importance of research on creep of wood and it should not be discriminated or neglected. To be able to understand and numerically or mathematically describe the rheological behavior of wood at various time periods, a wide range of experimental data not only on creep but also on stress relaxation is needed. Accounting for a broad spectrum of work on stress relaxation of wood, some interesting studies have been noticed. Stress relaxation experiments in bending were performed by Gaff and Gašparík (2015) on beech wood, by Kurenuma and Nakano (2012) on wet wood and by Tanimoto and Nakano (2012) on treated wood using aqueous alkali solution. Tensile stress relaxation tests of impregnated wood with water-based preservative at various temperatures were carried out by $\mathrm{Yu}$ et al. (2010). Tensile stress relaxation of pine wood exposed to relative humidity and temperature variations is studied by Li et al. (2012). Stress relaxation on microlevel of three different coniferous woods under tension parallel and compression perpendicular to the grain was analyzed by Kirbach et al. (1976). When it comes to stress relaxation of wood in constant environment, the available experimental studies are even more narrowed. Saifouni et al. (2016) analyzed stress relaxation in tension of silver fir in constant climate. Kubat and Klason (1991) reported 
experimental results of stress relaxation of Scots pine veneer at different stress levels and constant relative humidity. Additionally, Saifouni et al. (2016) and Kubat et al. (1989) presented the results of stress relaxation of wood exposed to changing relative humidity. A very extensive experimental study on stress relaxation of six tropical wood species at several levels of strain in compression and tension was carried out by Echeniques-Manrique (1969). Commonly, the researchers applied linear viscoelastic 'spring-dashpot' models to the experimental results at low strain levels. The abilities and limitations of one-dimensional (1D) 'spring-dashpot' models for predicting nonlinear time-dependent curves were theoretically discussed in Tschoegl (1989) and Findley et al. (1976). Practically, Echeniques-Manrique (1969) showed that $1 \mathrm{D} \mathrm{KD}$ model, also known as Burgers model, was unable to predict stress relaxation of different wood species under instant step excitation. Additionally, he claimed that nonlinear dashpot in series with 'Kelvin solid' fitted experimental data from stress relaxation much better. Mathematical formulation of the nonlinear dashpot was based on the 1D molecular deformation kinetics theory of flow processes (e.g., Krausz and Eyring 1975). A series of nonlinear dashpots in combination with other rheological assemblies was typically used for modeling creep behavior of wood (Caulfield 1985; Van der Put 1989; Hanhijärvi 1995a, b; Engelund and Svensson 2011). To the authors' best knowledge, the theory of deformation kinetics has not been applied to modeling rheological behavior of wood in more than one dimension so far. Rawat et al. (1998) used chemical kinetics to study the stress relaxation of pine wood blocks compressed parallel to the grain at constant relative humidity and room temperature.

With the aim of better understanding time-dependent behavior of wood and of offering fresh and up-to-date experimental data on stress relaxation in constant climate, this paper presents a new set of experimental results of stress relaxation of pine wood under compression parallel and perpendicular to the grain. Different levels of deformation were applied to the rectangular block specimens in consecutive manner. Additionally, tests until failure with constant deformation rate were performed. Strains in two perpendicular directions and load were monitored during the tests. Elastic moduli and Poisson's ratios were determined from tests with constant deformation rate. Similar to elastic material parameters, an attempt to find a set of viscoelastic material parameters that characterize the rheological behavior of wood was made. Hence, three two-dimensional (2D) linear viscoelastic models including different numbers of viscoelastic material parameters were calibrated to experimentally determined stress-time curves in the range of elastic strain.

\section{Materials and methods}

Experiments were performed by means of a uniaxial testing machine MTS ${ }^{\circledR}$. A force transducer LPS.105 of the capacity $100 \mathrm{kN}$ was used. During testing, force and axial and transverse displacements were measured on a front surface of the specimens. Force monitored with the load cell was exported to MTS TestSuite ${ }^{\mathrm{TM}}$ software, while the strain measurements were calculated and collected from compatible software, Advantage Video Extensometer (AVX04). All the measurements were 
exported with the frequency $10 \mathrm{~s}^{-1}$. The AVX software operated the digital camera, GigE with resolution of $1388 \times 1038$ pixel recording 17 frames per second. A material testing lens was used with magnification 0.2 and working distance $297 \mathrm{~mm}$. The software works on a principle of digital non-contact strain gauges projected on the image of the specimen's surface. The virtual strain gauge follows the displacements of two points that are physically marked on the specimen's surface. The AVX measurements meet the accuracy requirements for ASTM E83 Class B1 and ISO 9513 Class 0.5 calibration standards (MTS ${ }^{\circledR} 2017$ ). The advantages of measuring strain with digital method compared to a conventional method based on a comparative analysis of elastic constants determined with different methods were discussed in Crespo et al. (2017). In the experimental analyses at hand, 3 virtual strain gauges were placed in horizontal and 3 in vertical direction on the front surface of each tested specimen. The length of strain gauges in horizontal direction was $20 \mathrm{~mm}$ and in vertical direction $16 \mathrm{~mm}$. One strain gauge was placed in the center line and two others symmetrically by 10 and $8 \mathrm{~mm}$ offset in horizontal and vertical directions, respectively.

The experiments were carried out on Scots pine (Pinus sylvestris L.) grown in southern Sweden. During testing, climate was held constant at a temperature $T=20{ }^{\circ} \mathrm{C}$ and relative humidity $\mathrm{RH}=30 \%$. The used wood did neither contain knots, resin nor reaction wood. After the specimens had been cut into rectangular blocks with fairly smooth surfaces, they were conditioned to the target constant environment resulting in equilibrium moisture content $u=9 \%$. Their average crosssectional area was $A=5.915 \mathrm{~cm}^{2}$, height $h=3.95 \mathrm{~cm}$ and density $\rho=537 \mathrm{~kg} / \mathrm{m}^{3}$. Two batches of specimens were cut: The first batch consisted of 17 specimens with the grain aligned in the direction of deformation, and the second one contained 18 specimens with the grain perpendicular to the applied deformation. Therefore, the first batch was tested in compression in longitudinal (L) direction and the second one in transverse $(\mathrm{T})$ direction. Six specimens of each batch were deformed until failure with the constant rate $0.2 \mathrm{~mm} / \mathrm{min}$ in $\mathrm{L}$ and $0.6 \mathrm{~mm} / \mathrm{min}$ in $\mathrm{T}$ direction. The deformation rates were chosen so that the first peak compressive load in $\mathrm{L}$ direction was reached at approximately $5 \mathrm{~min}$ and the peak compressive load in $\mathrm{T}$ direction at approximately $2.5 \mathrm{~min}$. Several studies on the influence of loading rate on mechanical properties of wood were reported (Büyüksari 2017; Green et al. 1999; Gerhards 1977). Generally, it has been concluded that the strength and modulus of elasticity parallel to the grain decrease with decreasing loading rate. However, the influence of loading (or deformation) rate on mechanical properties of wood was not specifically addressed in this work.

The remaining specimens of each batch were tested under a stepwise deformation-controlled procedure. The deformation was controlled by a crosshead movement of the testing machine. The stepwise procedure consisted of different numbers of periods with constant deformation, i.e., relaxation periods, $t_{\text {relax }}$, that were interrupted by so-called active deformation periods where the deformation was applied with constant rate. The deformation rates were equal to those used in the experiments with constant deformation rate until failure. First, all specimens were stepwise deformed for 0.1 up to $0.6 \mathrm{~mm}$ which sums up to 6 deformation periods. Next, the specimens were deformed by steps of 0.15 or $0.3 \mathrm{~mm}$ of crosshead movement. After 
each active deformation period, the deformation was held constant for $t_{\text {relax }}=5 \mathrm{~min}$ for 8 specimens and $t_{\text {relax }}=60$ min for 3 specimens in L direction. In T direction, 8 specimens underwent the relaxation periods of $5 \mathrm{~min}$ and 4 specimens of $60 \mathrm{~min}$. Correspondingly, the specimens were sorted in 5 groups, which is summarized in Table 1. Since the specimens in groups L51 and L52 had the same testing protocol for the first six steps of active deformation and relaxation periods, they will be designated as group L5 for convenience.

The stress relaxation experiments with 5- and 60-min relaxation periods were carried out with the intention to elucidate and analyze in detail the short-term rheological behavior of wood subjected to the repetitive stepwise deformation. The influence of applying repetitive deformation steps, their magnitudes and the duration of relaxation periods on stress relaxation was investigated. The obtained experimental results present a fundamental basis in development of rheological models for wood material. With the acquired numerical values of material parameters, a direct application of the model to predicting long-term behavior of timber structures in constant climate is possible.

Stress retention and relaxation over particular relaxation period at certain deformation level were determined as follows

$$
\begin{aligned}
& \text { Stress retention }[/]=\frac{\sigma(t)}{\text { Maximum stress }} \\
& \text { Stress relaxation }[\%]=\left(1-\frac{\sigma(t)}{\text { Maximum stress }}\right) \times 100, \\
& \text { where } \sigma(t) \quad \text { was determined as ratio between compression force at time } t \\
& \left(0 \leq t \leq t_{\text {relax }}\right) \text { and initial cross-sectional area }(A) \text { of a specimen. 'Maximum stress' } \\
& \text { denoted the absolute value of the stress just before the beginning of each relaxation } \\
& \text { period. Figure } 1 \text { shows stress development and crosshead movement with time in } \\
& \text { stepwise deformation-controlled (crosshead movement) experiments for wood under }
\end{aligned}
$$
compression.

Table 1 Testing procedure of deformation-controlled relaxation under compression

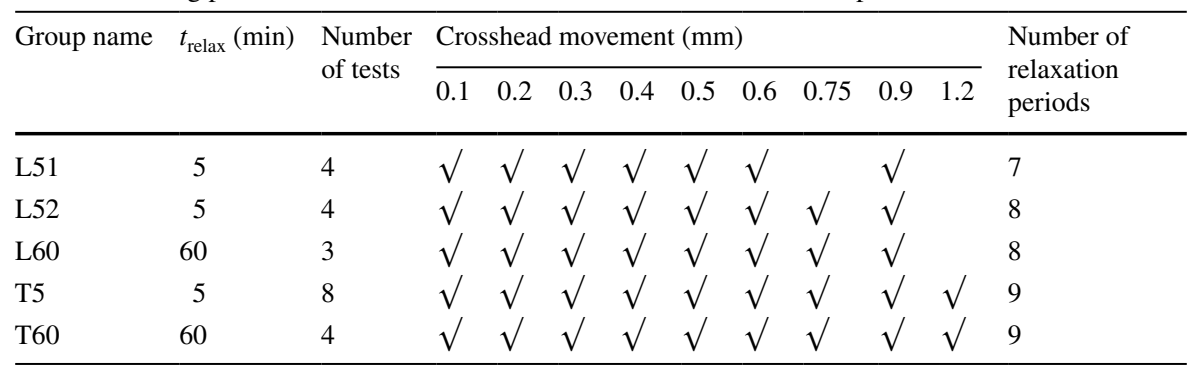

Group L5 = L51 + L52 up to $0.6 \mathrm{~mm}$ crosshead movement and 6 relaxation periods 
Fig. 1 Typical stress-time and stress-deformation curves obtained in deformation-controlled stress relaxation experiments for wood under compression in constant environment

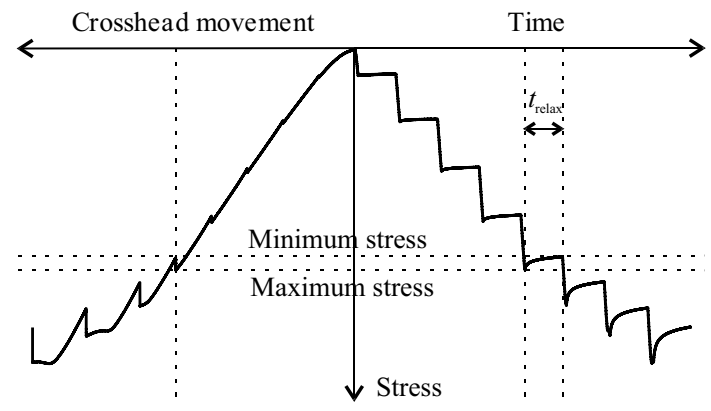

Determination of elastic modulus for each specimen is based on the mean values of the three measurements of strain in the direction of deformation, $\varepsilon_{i}$, and the corresponding stress, $\sigma_{i}$. The interval for determination of elastic modulus is chosen from 15 to $35 \%$ of ultimate compression stress, $\sigma_{\mathrm{u}}$, of each specimen. The ultimate compression stress is calculated as ratio between measured maximum compression force and initial cross-sectional area of a specimen $(A)$. The elastic modulus of particular specimen is determined by the equation

$$
E_{i}=\Delta \sigma_{i} / \Delta \varepsilon_{i}
$$

where $\Delta \sigma_{i}$ is an increment of stress and $\Delta \varepsilon_{i}$ is an increment of strain on the chosen interval. Poisson's ratio is calculated at $0.35 \times \sigma_{\mathrm{u}}$ for each specimen as

$$
v_{i j}=-\varepsilon_{j} / \varepsilon_{i},
$$

where $\varepsilon_{j}$ is the measured strain in the perpendicular direction. In accordance with material orthotropic directions, $i$ and $j$ stand for $\mathrm{L}$ or $\mathrm{T}$.

A coupled 2D formulation for modeling a material's viscoelastic response is used for predicting the obtained experimental results of stress relaxation in the range of elastic strain. A 2D mathematical formulation of a linear viscoelastic model based on a 'Kelvin solid' was first presented by Frandsen (2007). The formulation assumes material orthotropy and is composed of springs and dashpots that characterize linear elastic and viscous response of a material, respectively. Their combination in parallel enables nonlinear strain or stress development over time whether a constant stress or strain is prescribed. Even though the model is capable of predicting the nonlinear creep or relaxation behavior, the constitutive equations are linear elastic and linear viscoelastic. The original 2D Kelvin model (K) was upgraded with additional Kelvin element (KK model) and dashpot (KD model) in series by Huč and Svensson (2018). Governing constitutive equations of the three rheological models for wood in constant conditions are as follows

$$
\left[\begin{array}{c}
\sigma_{\mathrm{L}} \\
\sigma_{\mathrm{T}}
\end{array}\right]=\left[\begin{array}{ll}
Q_{\mathrm{LL}}^{0} & Q_{\mathrm{LT}}^{0} \\
Q_{\mathrm{TL}}^{0} & Q_{\mathrm{TT}}^{0}
\end{array}\right]\left[\begin{array}{c}
\varepsilon_{\mathrm{L}}^{0} \\
\varepsilon_{\mathrm{T}}^{0}
\end{array}\right],
$$




$$
\begin{gathered}
{\left[\begin{array}{c}
\sigma_{\mathrm{L}} \\
\sigma_{\mathrm{T}}
\end{array}\right]=\left[\begin{array}{ll}
Q_{\mathrm{LL}}^{i} & Q_{\mathrm{LT}}^{i} \\
Q_{\mathrm{TL}}^{i} & Q_{\mathrm{TT}}^{i}
\end{array}\right]\left[\begin{array}{c}
\varepsilon_{\mathrm{L}}^{i} \\
\varepsilon_{\mathrm{T}}^{i}
\end{array}\right]+\left[\begin{array}{cc}
\eta_{\mathrm{LL}}^{i} & \eta_{\mathrm{LT}}^{i} \\
\eta_{\mathrm{TL}}^{i} & \eta_{\mathrm{TT}}^{i}
\end{array}\right]\left[\begin{array}{c}
\dot{\varepsilon}_{\mathrm{L}}^{i} \\
\dot{\varepsilon}_{\mathrm{T}}^{i}
\end{array}\right], \quad \text { for } i=1, \quad \text { or } i=1 \quad \text { and } 2} \\
{\left[\begin{array}{c}
\sigma_{\mathrm{L}} \\
\sigma_{\mathrm{T}}
\end{array}\right]=\left[\begin{array}{cc}
\eta_{\mathrm{LL}}^{2} & \eta_{\mathrm{LT}}^{2} \\
\eta_{\mathrm{TL}}^{2} & \eta_{\mathrm{TT}}^{2}
\end{array}\right]\left[\begin{array}{c}
\dot{\varepsilon}_{\mathrm{L}}^{2} \\
\dot{\varepsilon}_{\mathrm{T}}^{2}
\end{array}\right]}
\end{gathered}
$$

where $(\cdot)$ denotes time derivative, $\sigma$ the stress, $\varepsilon^{0}$ the elastic strain, $\varepsilon^{1}$ and $\varepsilon^{2}$ the viscoelastic strains, and $Q$ and $\eta$ the material parameters. The total strains in $\mathrm{L}$ and T directions, $\varepsilon_{\mathrm{L}}$ and $\varepsilon_{\mathrm{T}}$, respectively, are assumed to be a sum of elastic and viscoelastic parts

$$
\left[\begin{array}{l}
\varepsilon_{\mathrm{L}} \\
\varepsilon_{\mathrm{T}}
\end{array}\right]=\sum_{i=0}^{2}\left[\begin{array}{c}
\varepsilon_{\mathrm{L}}^{i} \\
\varepsilon_{\mathrm{T}}^{i}
\end{array}\right],
$$

where $i=0$ is the elastic part and $i=1$ and/or 2 the viscoelastic parts. The elastic response is taken into account by Eq. (5) where the material parameters are determined from relations of elastic moduli and Poisson's ratios for orthotropic material. The viscoelastic response is included by Eq. (6) for $i=1$ in K model, by Eqs. (6) for $i=1$ and (7) in KD model and by Eqs. (6) for $i=1$ and 2 in KK model. The $\boldsymbol{Q}$ and $\boldsymbol{\eta}$ matrices in Eqs. (5)-(7) are symmetric; hence, $Q_{\mathrm{TL}}^{i}=Q_{\mathrm{LT}}^{i}$ and $\eta_{\mathrm{TL}}^{i}=\eta_{\mathrm{LT}}^{i}$ for $i=1$, 2 and 3. The rheological models K, KD and KK consist of 3 elastic and 6, 9 and 12 different viscoelastic material parameters, respectively, that require to be calibrated against experimental results. The $2 \mathrm{D}$ rheological models were successfully applied to experimental results of viscoelastic creep of wood in Kawahara et al. (2015) and Huč and Svensson (2018). In this study, an attempt of the models' application on the stress relaxation of wood is made.

\section{Results and discussion}

\section{Experimental results}

Table 2 shows mean value, standard deviation (std), coefficient of variation (cv), maximum (max) and minimum (min) values of the elastic moduli for specimens

Table 2 Elastic moduli, Poisson's ratios and ultimate compressive strength for Scots pine

\begin{tabular}{lllllll}
\hline & $E_{\mathrm{L}}(\mathrm{MPa})$ & $E_{\mathrm{T}}(\mathrm{MPa})$ & $\sigma_{\mathrm{u}, \mathrm{L}}(\mathrm{MPa})$ & $\sigma_{\mathrm{u}, \mathrm{T}}(\mathrm{MPa})$ & $\nu_{\mathrm{LT}}(/)$ & $\nu_{\mathrm{TL}}(/)$ \\
\hline Min & 15,719 & 530 & 50.5 & 7.0 & 0.09 & 0.009 \\
Max & 19,353 & 663 & 59.1 & 7.8 & 0.49 & 0.023 \\
Mean & 17,654 & 599 & 56.4 & 7.6 & 0.33 & 0.016 \\
Std & 1257 & 56 & 3.0 & 0.3 & 0.16 & 0.006 \\
$\mathrm{cv}(\%)$ & 7.1 & 9.4 & 5.4 & 4.1 & 48.7 & 36.6 \\
\hline
\end{tabular}


tested in $\mathrm{L}$ and $\mathrm{T}$ directions. Corresponding values for Poisson's ratios and ultimate compression stresses are also tabulated.

Material properties of the tested specimens are in the expected range, if compared to the values in the literature for pine (Kollman and Côté 1968). Lower values of elastic modulus in compression parallel to the grain for species of pine wood were reported in Aira et al. (2014), Baltrušaitis and Aleinikovas (2012) and Kretschmann (2010). However, Jin-Kyu et al. (2007) and Aira et al. (2014) discussed possible reasons for discrepancies among the experimental data from different analyses, such as high probability of slight variations in experimental conditions, i.e., RH, deformation rate and the wood itself, i.e., density, degree of growth and imperfections, specimen shape or different methods used for evaluating the elastic material parameters. The experimentally determined Poisson's ratios have high coefficients of variation. Beside the material behavior, in particular the uncertainty of strain measurements perpendicular to the applied deformation is most probably a reason for large coefficients of variation of Poisson's ratios.

Figure 2 shows stress retention curves for $t_{\text {relax }}=60$ min under applied stepwise deformation in (a) $\mathrm{L}$ and (b) $\mathrm{T}$ directions. The presented stress retention curves are determined as average among the specimens in groups L60 and T60. Stress retention is a nonlinear function of time at all deformation levels. Magnitude of the stress retention curves varies with the applied deformation levels. The shape of stress retention curves over time for specimens in groups L5 and T5 is very similar to those in Fig. 2a, b, respectively.

Figure 3 depicts stress relaxation at the end of each relaxation period in relation to maximum stress reached just before the start of each relaxation period for all tested groups. Markers designate mean value and bars designate minimum and maximum values of depicted variables. Mean values of stress relaxation plotted in relation to maximum stresses for the applied deformation levels show a convex function formation. First, stress relaxation decreases to a certain deformation level, where the trend turns and stress relaxation starts increasing. Meanwhile, the maximum stress
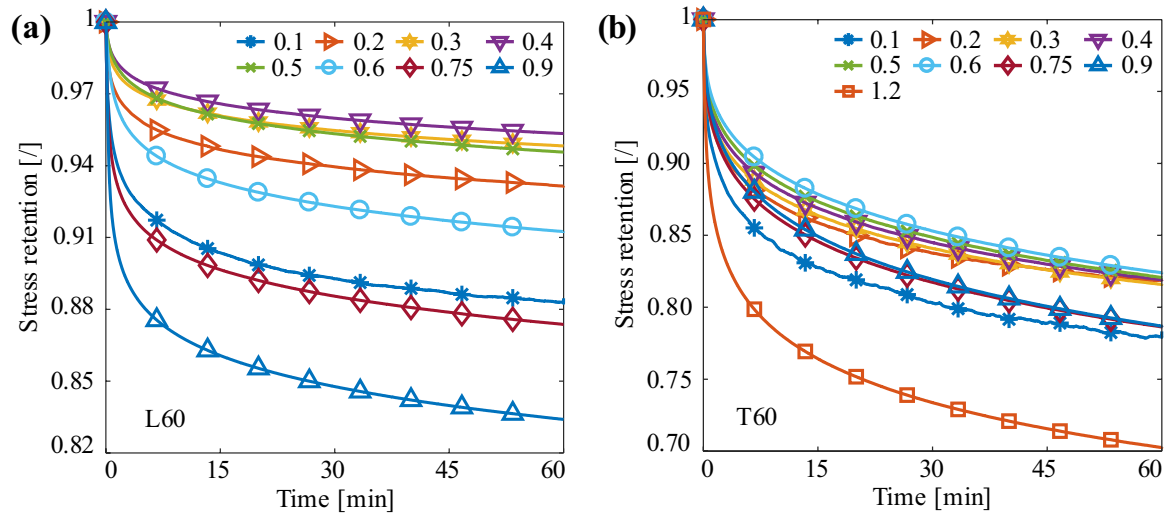

Fig. 2 Stress retention curves for tested groups a L60 and b T60 under applied consecutive deformation levels 

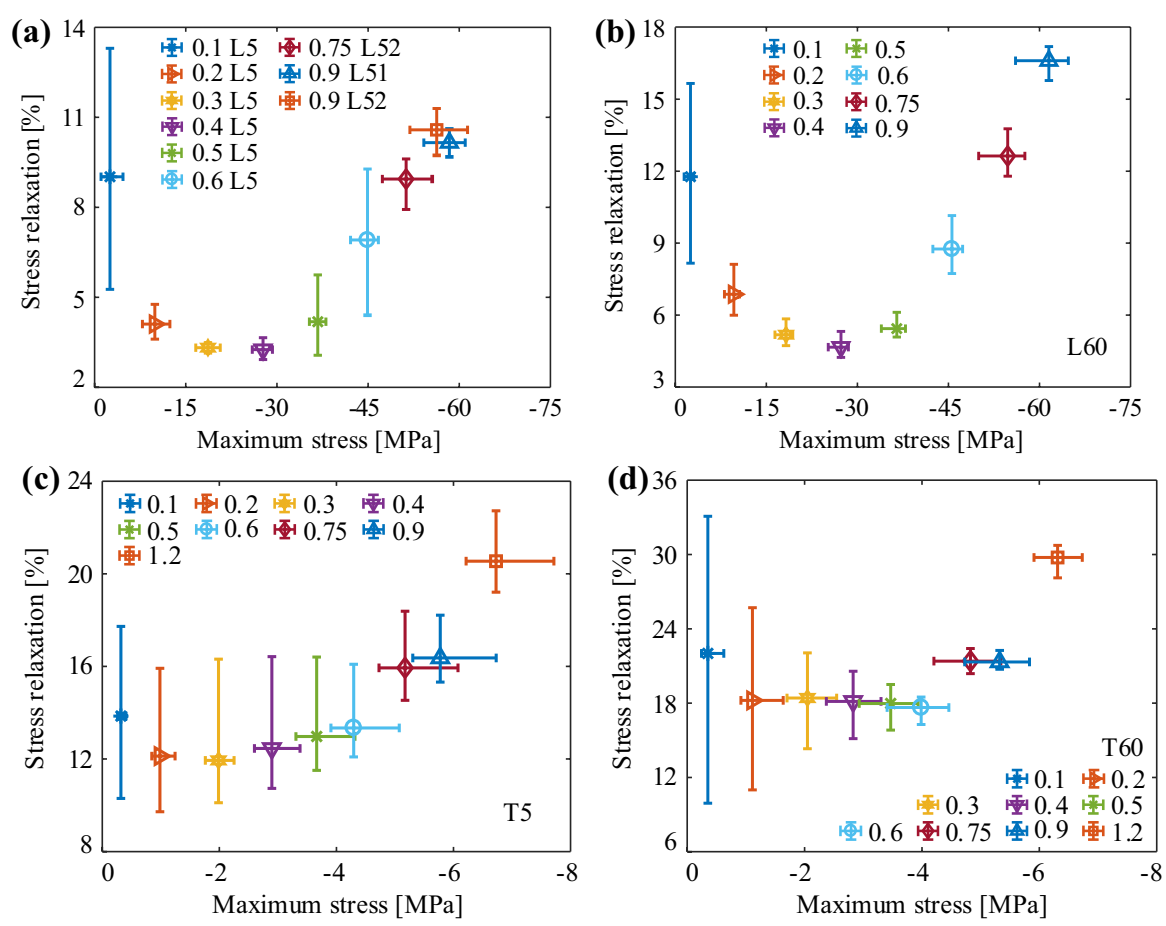

Fig. 3 Stress relaxation at the end of each relaxation period corresponding to applied deformation levels in compression in longitudinal direction a L5, b L60, and in transverse direction c T5, d T60

is monotonically increasing. Obviously, the material behaves differently under low deformation than under high deformation. Probably, the change in material's behavior appears in the transition from recoverable to permanent deformation. In the range of recoverable elastic deformation, each additional deformation step decreases the stress relaxation, while in the range of irrecoverable plastic deformation the opposite trend is observed, which is in agreement with the experimental results of stress relaxation in compression reported by Echeniques-Manrique (1969). For specimens deformed in L direction (Fig. 3a, b), the elastic limit is reached between deformation levels 0.4 and $0.5 \mathrm{~mm}$. The results in Fig. 3c, d indicate the limit of elasticity is between deformation levels 0.3 and $0.4 \mathrm{~mm}$ for specimens in group $\mathrm{T} 5$ and between 0.6 and $0.75 \mathrm{~mm}$ for group T60. In general, the difference in mean values of stress relaxation decreases the closer to the limit of elasticity the material is deformed. The variation of mean value of stress relaxation is about $2 \%$ for deformation levels $0.3-0.5 \mathrm{~mm}$.

It must be noted that the results regarding the deformation levels 0.1 and $0.2 \mathrm{~mm}$ of crosshead movement are noisier in comparison with the results of consecutive deformation levels. Additionally, the scatter of stress relaxation at deformation level $0.1 \mathrm{~mm}$ is the highest (Fig. 3). The noise and scatter of the results are not necessarily related to the material behavior, but could be affected by uncertainty of the used equipment in the range of recoverable strains. Scatter of the maximum stresses 
is relatively small and quite insignificant; however, it shows a tendency to increase with increasing applied deformation. The mean values of stress relaxation at the end of the relaxation periods are about $40 \%$ higher on average in groups L60 and T60 than in L5 and T5, respectively. In general, the stress relaxation is higher when the material is deformed in $\mathrm{T}$ direction compared to $\mathrm{L}$ direction.

Relative error defined as a difference between the maximum stresses in groups L5 and L60, normalized by the maximum stress of group L5 for particular deformation level tends to slightly increase with increasing maximum stress but is never higher than $9 \%$. This could indicate that the deformation history with longer relaxation periods results in higher maximum stresses reached in the range of irrecoverable strain. The mean maximum stress at deformation level $0.9 \mathrm{~mm}$ is a bit lower (approx. 4\%) in group L52 with a total of 8 relaxation periods than in group L51 which was subjected to one relaxation period less (Fig. 3a). Since the minimum and maximum values of stress relaxation and maximum stress of group L52 envelop the results of group L51 at deformation level $0.9 \mathrm{~mm}$, the additional relaxation period seems not to influence the magnitude of the maximum stresses reached in the range of irrecoverable strain. Relative comparison of maximum stresses for specimens deformed stepwise in $\mathrm{T}$ direction, groups $\mathrm{T} 5$ and T60, does not confirm the influence of the duration of relaxation periods on the magnitude of maximum stress reached at each deformation level since the relative error is spread disorderly and is not higher than $8 \%$. Analyzing the mean values of stress relaxation at time $5 \mathrm{~min}$ of each deformation period in all tested groups shows that they are on average $15 \%$ higher in group L5 compared to group L60 and 27\% higher in group T5 compared to group T60. This indicates a considerable influence of the duration of relaxation period on the stress relaxation.

\section{Modeling}

The governing Eqs. (5)-(8) of the three viscoelastic models (K, KD, KK) are implemented in a finite element software. Geometry corresponds to the actual experimental data. The elastic material parameters are accounted for as determined from the tests with constant deformation rate (Table 2). When the applied crosshead movement and the deformation determined as a product of specimen height and the measured strain in the direction of compression are compared, a significant difference is noticed. The loss of deformation occurred due to the friction on the contact surfaces between machine's steel loading plates and the specimen. The amount of lost deformation is quite high, especially in compression along the grain. In order to simulate actual strain-stress state in recoverable range, an average of measured strain in the direction of compression is taken as an input in the numerical models. The strain input for groups L60 $\left(\varepsilon_{\mathrm{L}}\right)$ and T60 $\left(\varepsilon_{\mathrm{T}}\right)$ is depicted in Fig. 4a. It corresponds to deformation levels named $0.1-0.4$ and $0.1-0.5$ for $\mathrm{L}$ and $\mathrm{T}$ directions, respectively, and is associated with crosshead movement in $\mathrm{mm} .4$ and 5 deformation steps are modeled for the applied deformation in $\mathrm{L}$ and $\mathrm{T}$ directions, respectively. Further deformation steps are considered to induce permanent deformation since the stresses exceeded $50 \%$ of mean value of ultimate compression stress. The unknown 

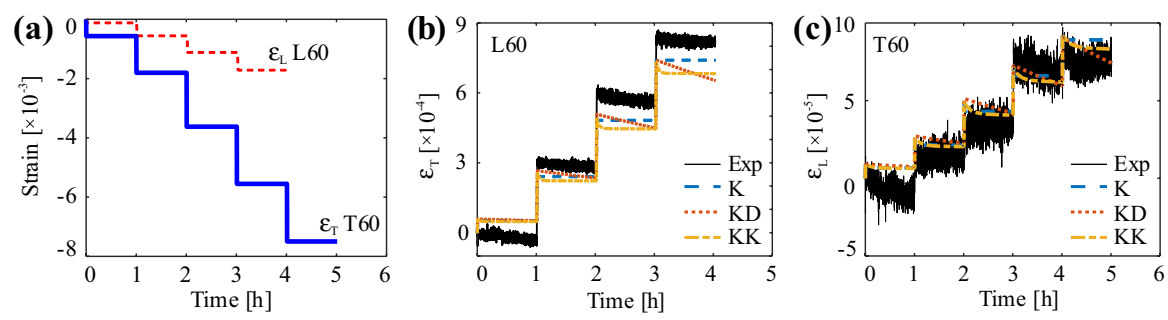

Fig. 4 a Strain input in the direction of applied deformation in numerical models for groups L60 and T60; measured (Exp) and calculated (K, KD, KK models) strain perpendicular to the applied deformation for groups b L60 and $\mathbf{c}$ T60

viscoelastic material parameters of each model are determined by least squares fitting procedure of numerically predicted against experimentally obtained stress-time curves for $\mathrm{L}$ and $\mathrm{T}$ directions simultaneously. To obtain only one targeted experimental stress-time curve per tested group, the average of the ratio between measured force and initial cross-sectional area of each specimen is taken at all measured times. The fitting procedure is applied to 5 points of each relaxation period, i.e., minimum and maximum value, and 1/72, 1/24 and 1/2 of $t_{\text {relax }}$. Figure 5 depicts numerically obtained stress curves by $\mathrm{K}, \mathrm{KD}$ and $\mathrm{KK}$ models together with experimental curves (Exp) for groups L60 and T60. Figure 4b, c shows the corresponding measured and modeled strains perpendicular to the applied deformation for groups L60 and T60, respectively. The experimental data are quite scattered and uncertain; however, the modeled strains are more or less of the same magnitude as experimental data. Numerical results of the three viscoelastic models calibrated to experimental results L5 and T5 are not shown in the paper since their predictions were very similar to those of groups L60 and T60.

Doubtlessly, wood material behaves non-linearly viscoelastic under high deformation or stress. At low strain and stress levels, its behavior is also nonlinear
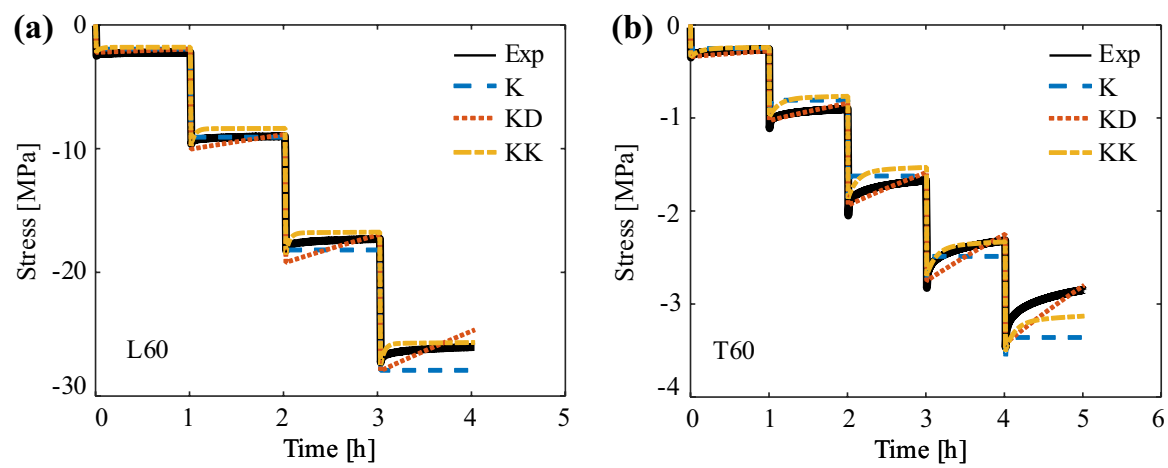

Fig. 5 Experimental (Exp) and numerically predicted (K, KD, KK) stress-time curves for applied deformation in a longitudinal (L60) and $\mathbf{b}$ transverse directions (T60) simultaneously 
viscoelastic but less prominent that allows for the assumption of linear viscoelasticity to be a good approximation (Morlier and Palka 1994). This was confirmed with the experimental results presented in this paper as well as in numerous other experimental studies (e.g., Echeniques-Manrique 1969; Schniewind and Barrett 1972; Hanhijärvi 1999; Hunt 1999). The assumption of linear viscoelasticity at low strain or stress levels of wood is especially convenient when predicting material behavior by means of linear 'spring-dashpot' models. Excluding the non-linearity and plasticity, the viscoelastic modeling becomes simpler but limited to low, recoverable stress-strain levels. Yet, the linear viscoelastic models usually include a number of material parameters that do not have real physical meaning and have to be determined by curve fitting procedure. Theoretically, a distinctive feature of linear viscoelasticity is the Boltzmann superposition principle which states for stress relaxation that the stress at current time is a sum or integral or linear superposition of the stresses at previous times obtained under arbitrary strain history (Tschoegl 1989). For Boltzmann superposition to hold an impulse or instantaneous step, excitations, like delta function, have to be applied. In cases such as the stress relaxation experiments performed where the deformation is applied stepwise, the Boltzmann superposition is not so straightforward in a sense that the normalized stress retention curves do not overlay each other. Instead the stress retention over time is decreasing with additional deformation steps in the range of elastic strain. That was observed in the experimental results and confirmed by the $2 \mathrm{D}$ linear viscoelastic models $\mathrm{K}, \mathrm{KD}$ and KK. The difference between stress retention curves at consecutive deformation levels implies the amount of stress relaxation that occurred during the active deformation periods. In other words, a portion of viscoelastic strains developed not only at constant deformation but also during the active deformation periods. Figure 6 shows the normalized stress retention curves for groups L60 and T60 obtained from experimental data (also shown in Fig. 2) and the KK model. It can be seen that the difference between stress retention curves is decreasing with increasing deformation levels in the range of recoverable elastic strains. The KK model is able to predict the decreasing difference but not in the same amount as shown by the experiments.
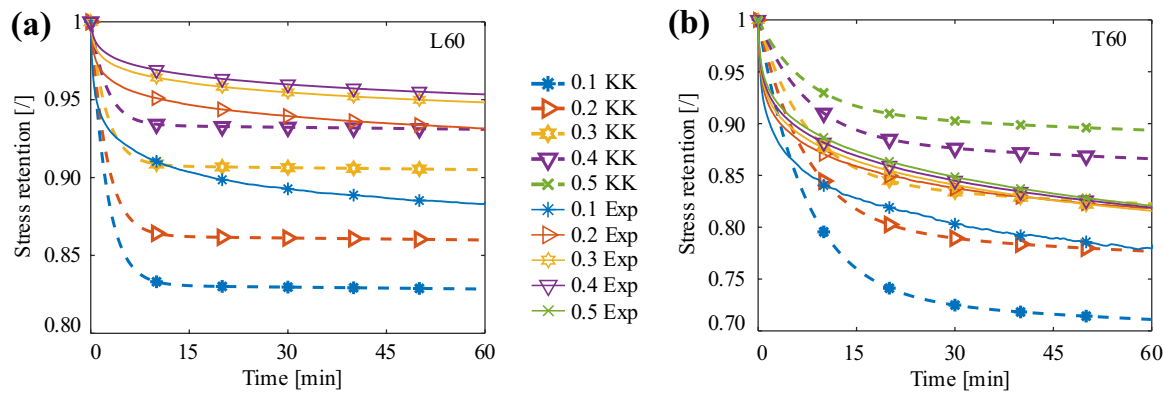

Fig. 6 Stress retention curves determined from experimental data (Exp) and predicted by the linear viscoelastic model (KK, dashed lines) for consecutive deformation steps in a longitudinal (L60) and $\mathbf{b}$ transverse directions (T60) simultaneously 
Consequently, the numerically predicted stress retention curves of consecutive deformation steps deviate more markedly.

Generally, it can be concluded that the $\mathrm{K}$ model is not very successful in predicting experimental stress-time curves. Moreover, the ability of the $\mathrm{K}$ model to predict normalized stress retention curves for each relaxation period is practically nonexistent in L direction and substantially too low in $\mathrm{T}$ direction. The KD model is almost not able to produce nonlinear stress relaxation behavior, as similarly argued by Echeniques-Manrique (1969), while the minimum and maximum stresses of each relaxation period fit quite well (Fig. 5). Stress retention curves corresponding to stress-time predictions of $\mathrm{K}$ and $\mathrm{KD}$ models are not shown in detail in the paper due to the invaluable results. As expected, the stress-time curves and corresponding stress retention curves are best predicted by the KK model that has the largest number of viscoelastic material parameters among the used models. Less promising is the fact that the models are not able to predict viscoelastic behavior of the material in stepwise relaxation test of wood under compression as good as they are able to predict viscoelastic creep strain of different wood species under constant tension where the excitation is instant (Huč and Svensson 2018).

It should be noted that the fitted viscoelastic material parameters influence the shape and the magnitude of the predicted stress retention and stress-time curves greatly. Therefore, numerous solutions could be obtained by the same model depending on the values of the viscoelastic material parameters. For instance, the gap between the stress retention curves could be decreased or the shape of the curves could be more alike to experimental results. To the authors' best knowledge, both could not be obtained with the same set of viscoelastic material parameters. Applying more advanced or expert fitting curve methods could most probably enhance the obtained numerical fits. Likewise, numerical prediction of stress relaxation process of wood could be obtained by adding more viscoelastic elements such as 'Kelvin solid' in series to KK model. Values of the viscoelastic material parameters differ among the models; in addition, it is not necessary that the viscoelastic material parameters obtained for groups L60 and T60 would also give the best fits of experimental results L5 and T5.

\section{Conclusion}

Scots pine specimens were tested in compression in the grain direction and perpendicular to the grain with constant deformation rate until failure and under stepwise deformation-controlled stress relaxation. The temperature around the specimen and its moisture content were held constant during testing. The stiffness and ultimate strength of the material were much higher in the direction of the grain than in the transverse direction. Corresponding Poisson's ratios were determined with large coefficients of variation. The stress relaxation experiments revealed that stress relaxation at the end of the relaxation periods was a nonlinear function of maximum compressive stresses reached before the start of the particular relaxation period. The stress retention was a nonlinear function of time for all deformation levels applied. The non-linearity was more prominent under higher deformation levels. The stress 
retention was gradually decreasing when material was deformed in the irreversible or plastic range of strain, while under low reversible strains the stress retention was increasing. The material experienced greater stress relaxation when deformed in the transverse than in the longitudinal direction.

Three linear rheological 2D models with different number of viscoelastic material parameters were used for predicting experimentally obtained stress curves in the range of recoverable strains. Elastic material parameters were used as determined in tests with constant deformation rate until failure. Viscoelastic material parameters were calibrated against experimental data. The same set of material parameters was used for predicting stress development over time in $\mathrm{L}$ and $\mathrm{T}$ directions. The input deformation protocol in the models was assigned according to the measured strain in the direction of applied deformation. The active deformation periods with nonzero deformation rate were also taken into account. The analyses showed that the simple viscoelastic $\mathrm{K}$ model was not capable to simulate stress relaxation. The numerical prediction was improved with additional viscoelastic material parameters that were built in the KK model. According to the KK model's results, it could be concluded that the material behavior observed by tests was very close to linear viscoelastic in relaxation under low deformation. Since the stress relaxation was taken into account not only in relaxation periods but also during the active deformation periods with nonzero deformation rate the stress retention curves were not superimposed. The difference between the curves obtained from experimental results was small if compared to the numerical predictions.

It was shown already the models were able to predict viscoelastic creep response in two perpendicular directions simultaneously when one load level was applied instantaneously. Analogously, it can be expected if only one level of deformation is applied instantaneously the tuning of the models to stress relaxation would also be satisfactory. As shown, already the simple K model was good enough in predicting viscoelastic creep response of wood under instantaneously applied constant load. This might not be the case when the prediction of viscoelastic behavior of wood in stress relaxation under instantaneous step deformation is to be desired. There, the models with higher number of viscoelastic material parameters (e.g., KK) might be required to obtain accurate fit to experimental results. If such analysis was carried out, it would be interesting to compare the numerical values of viscoelastic material parameters of the particular model calibrated to the stress relaxation and creep separately. Since they trigger the same molecular processes, it is expected that one set of viscoelastic material parameters will render equally good predictions of the viscoelastic response of wood in creep and stress relaxation. Yet, this hypothesis needs to be confirmed. Obviously, when a stepwise excitation is applied the material behavior becomes much harder to predict. The task is especially challenging when particular deformation or load level is studied in detail. In the future, the ability of the models to predict a stepwise viscoelastic creep would be interesting to investigate. The question arises whether the models will be as successful in predicting viscoelastic creep under stepwise loading as they were when the constant load was applied instantaneously. Afterward, the appropriate and more specific conclusions about the capability and suitability of the models for predicting viscoelastic behavior of wood under stepwise excitation can be drawn. In order to find better theoretical models for 
predicting the stress relaxation in wood material, it might also be worth working on a formulation including nonlinear dashpots in two orthotropic directions.

Acknowledgements The financial support by Gunnar Ivarson's Foundation (Gunnar Ivarsons Stiftelse för Hållbart Samhällsbyggande, GIS) made this work possible. The work of Tomaž Hozjan received support from the research core funding No. P2-0260 by the Slovenian Research Agency.

Open Access This article is distributed under the terms of the Creative Commons Attribution 4.0 International License (http://creativecommons.org/licenses/by/4.0/), which permits unrestricted use, distribution, and reproduction in any medium, provided you give appropriate credit to the original author(s) and the source, provide a link to the Creative Commons license, and indicate if changes were made.

\section{References}

Aira JR, Arriaga F, Iniguez-Gonzalez G (2014) Determination of elastic constants of Scots pine (Pinus sylvestris L.) wood by means of compression tests. Biosyst Eng 126:12-22

Baltrušaitis A, Aleinikovas M (2012) Early-stage prediction and modelling strength properties of lithuanian-grown scots pine (Pinus sylvestris L.). Balt For 18:327-333

Bažant ZP (1985) Constitutive equation of wood at variable humidity and temperature. Wood Sci Technol 19:159-177

Büyüksari Ü (2017) Effect of loading rate on mechanical properties of micro-size Scots Pine wood. BioResources 12:2721-2730

Caulfield DF (1985) A chemical kinetics approach to the duration-of-load problem in wood. Wood Fiber Sci 17:504-521

Crespo J, Aira JR, Vazquez C, Guaita M (2017) Comparative analysis of the elastic constants measured via conventional, ultrasound, and 3-D digital image correlation methods in Eucalyptus globulus Labill. BioResources 12:3728-3743

Echeniques-Manrique R (1969) Stress relaxation of wood at several levels of strain. Wood Sci Technol 3:49-73

Eitelberger J, Bader TK, de Borst K, Jäger A (2012) Multiscale prediction of visoelastic properties of softwood under constant climatic conditions. Comput Mater Sci 55:303-312

Engelund ET, Svensson S (2011) Modelling time-dependent behaviour of softwood using deformation kinetics. Holzforschung 65:231-237

Findley WN, Lai JS, Onaran K (1976) Creep and relaxation of nonlinear viscoelastic materials. Dover, New York

Flügge W (1967) Viscoelasticity. Blaisdell Publishing Company, University of Michigan, Michigan

Frandsen HL (2007) Selected constitutive models for simulating the hygromechanical response of wood. Dissertation, Aalborg University

Gaff M, Gašparík M (2015) Influence of cyclic stress on the relaxation speed of native and laminated wood. Bioresorces 10:402-411

Gerhards CC (1977) Effect of duration and rate of loading on strength of wood and wood-based materials. U.S. Department of Agriculture, Madison

Green DW, Winandy JE, Kretschmann DE (1999) Mechanical properties of wood. Forest Products Laboratory, Madison

Hanhijärvi A (1995a) Deformation kinetics based rheological model for the time-dependent and moisture induced deformation of wood. Wood Sci Technol 29:191-199

Hanhijärvi A (1995b) Modelling of creep deformation mechanisms in wood. Technical Research Centre of Finland, Espoo

Hanhijärvi A (1999) Deformation properties of Finnish spruce and pine wood in tangential and radial directions in association to high temperature drying, Part II. Experimental results under constant conditions (viscoelastic creep). Holz Roh- Werkst 57:365-372

Hanhijärvi A, Hunt D (1998) Experimental indication of interaction between viscoelastic and mechanosorptive creep. Wood Sci Technol 32:57-70

Huč S, Svensson S (2018) Coupled two-dimensional modelling of viscoelastic creep of wood. Wood Sci Technol 52(1):29-43 
Hunt DG (1999) A unified approach to creep of wood. Proc R Soc Lond A 455:4077-4095

Jin-Kyu S, Sun-Young K, Sang-Won O (2007) The compressive stress-strain relationship of timber. In: International conference on sustainable building Asia pp 977-982

Kawahara K, Ando K, Taniguchi Y (2015) Time dependence of Poisson's effect in wood IV: influence of grain angle. J Wood Sci 61:372-383

Kirbach E, Bach L, Wellwood RW, Wilson JW (1976) On the fractional stress relaxation of coniferous wood tissues. Wood Fiber 8:74-84

Kollman FFP, Côté WA Jr (1968) Principles of wood science and technology I, solid wood. Springer, Berlin

Krausz AS, Eyring H (1975) Deformation kinetics. Wiley, New York

Kretschmann DE (2010) Wood handbook: wood as an engineering material. Forest Products Laboratory, Madison

Kubat DG, Klason C (1991) Stress relaxation in wood (Scots pine veneer). Part II Quantitative comparison with the prediction of a cooperative flow model. J Mater Sci 26:5261-5268

Kubat DG, Samuelsson S, Klason C (1989) Stress relaxation in wood (Scots pine veneer). J Mater Sci 24:3541-3548

Kurenuma Y, Nakano T (2012) Analysis of stress relaxation on the basis of isolated relaxation spectrum for wet wood. J Mater Sci 47:4673-4679

Li XQ, Wang XM, Yu JF (2012) Research on tensile stress relaxation characteristics of Pinus sylvestris. Mater Sci Forum 704-705:480-485

Morlier P, Palka LC (1994) Modelling of time-dependent deformation. Creep in timber structures. E\&F, Spon, London

MTS $^{\circledR}$ (2017) Services \& accessories. https://www.mts.com/cs/groups/public/documents/library/ dev_003828.pdf. Accessed 25 Sept 2017

Navi P, Stanzl-Tschegg S (2009) Micromechanics of creep and relaxation of wood. A review. Holzforschung 63:186-198

Ożyhar T, Hering S, Niemz P (2013) Viscoelastic characterization of wood: time dependence of the orthotropic compliance in tension and compression. J Rheol 57:699-717

Rawat SPS, Breese MC, Khali DP (1998) Chemical kinetics of stress relaxation of compressed wood blocks. Wood Sci Technol 32:95-99

Saifouni O, Destrebecq JF, Froidevaux J, Navi P (2016) Experimental study of mechanosorptive behavior of softwood in relaxation. Wood Sci Technol 50:789-805

Schniewind AP, Barrett JD (1972) Wood as a linear viscoelastic material. Wood Sci Technol 6:43-57

Taniguchi Y, Ando K (2010) Time dependence of Poisson's effect in wood I: the lateral strain behavior. J Wood Sci 56:100-106

Tanimoto T, Nakano T (2012) Stress relaxation of wood partially non-crystallized using aqueous $\mathrm{NaOH}$ solutions. Carbohydr Polym 87:2145-2148

Tschoegl NW (1989) The phenomenological theory of linear viscoelastic behavior: an introduction. Springer, Berlin

Van der Put TACM (1989) Deformation and damage processes in wood. Delft University Press, Delft

Yu LL, Cao JZ, Zhao GJ (2010) Tensile stress relaxation of wood impregnated with different ACQ formulations at various temperatures. Holzforschung 64:111-117 